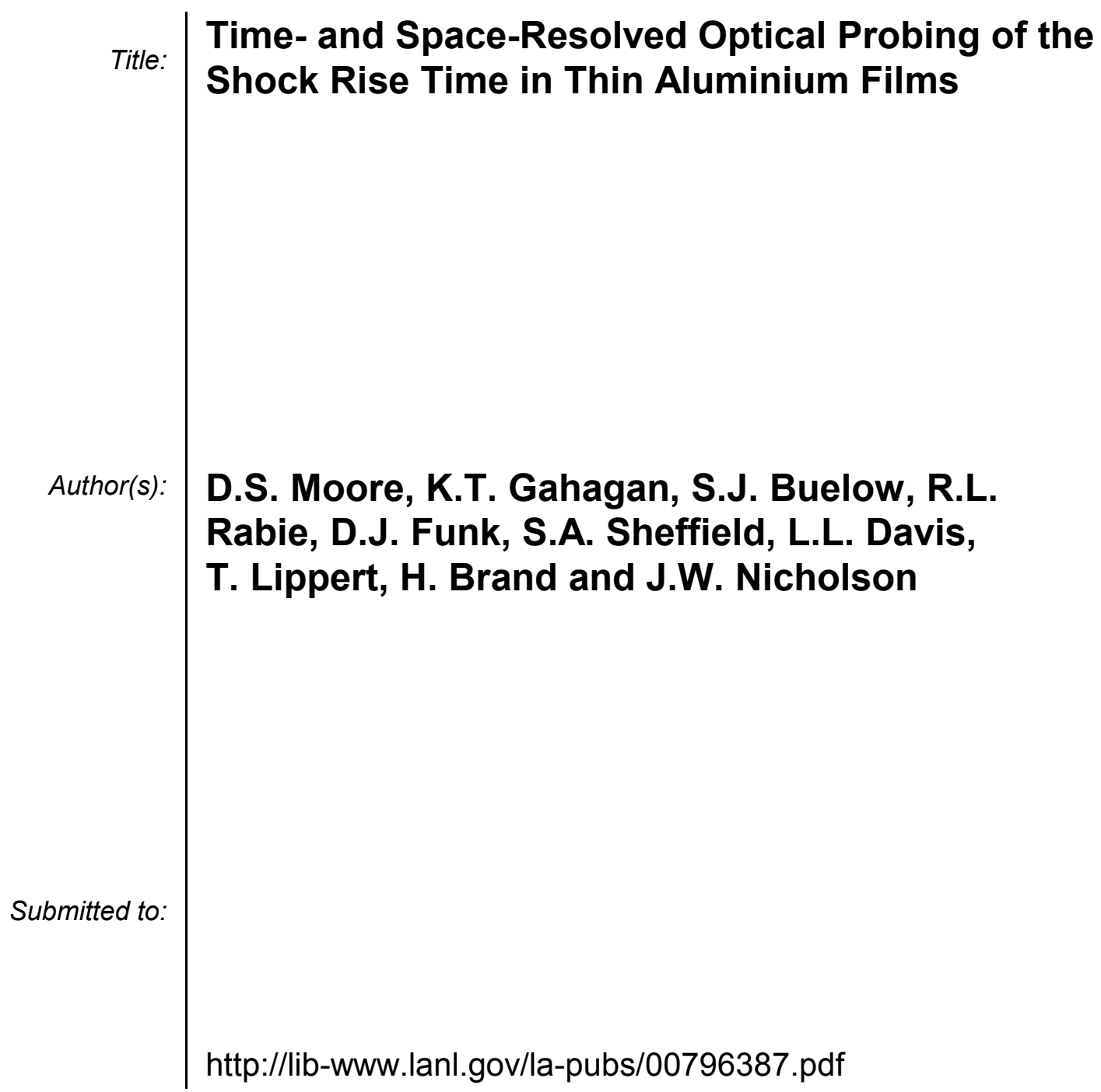




\title{
Time- and Space-Resolved Optical Probing of the Shock Rise Time in Thin Aluminium Films
}

\author{
D.S. Moore ${ }^{1}$, K.T. Gahagan ${ }^{2}$, S.J. Buelow ${ }^{2}$, R.L. Rabie ${ }^{1}$, D.J. Funk ${ }^{1}$, S.A. Sheffield ${ }^{1}$, \\ L.L. Davis ${ }^{1}$, T. Lippert ${ }^{3}$, H. Brand ${ }^{4}$, and J.W. Nicholson ${ }^{5}$ \\ ${ }^{1}$ Dynamic Experimentation Division, Los Alamos National Laboratory, Los Alamos, NM 87545 \\ ${ }^{2}$ Chemical Sciences and Technology Division, Los Alamos National Laboratory, Los Alamos, NM 87545 \\ ${ }^{3}$ Paul Scherrer Institute, CH-5232 Villigen PSI, Switzerland \\ ${ }^{4}$ Theoretical Division, Los Alamos National Laboratory, Los Alamos, NM 87545 \\ ${ }^{5}$ Phillips Laboratory, Kirtland Air Force Base, Albuquerque, NM
}

\begin{abstract}
The time resolution of most common methods (various forms of interferometry, e.g. VISAR, Fabry-Perot, ORVIS) used to measure the free surface particle velocity as a shock exits from a shocked material is from a few ns down to about $200 \mathrm{ps}$. Dlott et al. have recently obtained a shock rise time resolution of about 25 ps using a method involving singular value decomposition of coherent antiStokes Raman (CARS) spectra from thin film nanogauges. We are using frequency domain interferometric methods utilizing 120 fs pulses from a chirped-pulse amplified Ti:sapphire laser to measure the particle velocity rise time as a shock (driven by ca. $0.5 \mathrm{~mJ}$ pulses focussed to $75 \mu \mathrm{m}$ diameter) exits the free surface of 0.5-2 $\mu \mathrm{m}$ aluminium films on thin glass substrates, following the example of Evans, et al. Some preliminary results of these investigations are reported.
\end{abstract}

\section{INTRODUCTION}

Seemingly since the first cogitations about the chemical mechanism of detonation, methods have been developed with shorter and shorter time resolution to study the problem as well as the underlying fundamental question of the thickness of the detonation wave or shocks in general. In every case to date for experiments intended to measure the actual shock or free-surface velocity rise time, it has been found to be shorter than the time resolution of the probing method employed.

Currently, the most common methods utilized to measure the free surface particle velocity as a shock exits from a subject material are various forms of interferometry (VISAR (1), Fabry-Perot (2), ORVIS (3)) with time resolutions from a few ns down to about 200 ps (4). Dlott et al. $(5,6)$ have developed a novel method utilizing singular value decomposition of CARS spectra of laser-driven shocks in thin-layer anthracene nanogauges, and have measured a shock rise time of $<25 \mathrm{ps}$, which is the time resolution of their method.

A new spectroscopic method, frequency-domain interferometry $(7,8)$, has features that show promise for much shorter time resolution in these measurements. We have pursued using these interferometric methods to try to measure the free surface velocity rise time in thin aluminium films subjected to femtosecond laser-driven shocks.

\section{EXPERIMENTAL}

Thin aluminium films of varying thickness $(0.5$ to $2 \mu \mathrm{m}$ ) were vapor plated onto microscope slide cover slips. The shocks were driven into the aluminium films, in the manner of Evans et al. (9), 
by the deposition of laser light from a chirped pulse amplified Ti:sapphire laser. The pulses from this laser are ca. $120 \mathrm{fs}$ FWHM long at a wavelength of $798 \mathrm{~nm}$ with ca. $500 \mu \mathrm{J}$ of energy per pulse. The illuminated spot diameter is nominally $75 \mu \mathrm{m}$.

Frequency domain interferometry was performed using a probe pulse optically split from the shockdriving laser pulse. A Michelson interferometer with variable uneven leg length was then used to produce a pair of $120 \mathrm{fs}$ collinear probe pulses separated by variable times (4 to $16 \mathrm{ps).}$

The experimental arrangement is shown schematically in Fig. 1.

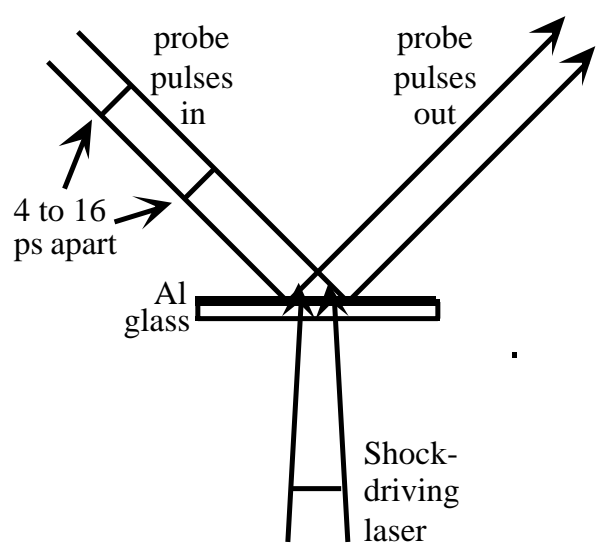

Figure 1. Schematic diagram of the femtosecond laser-driven shock/frequency domain interferometry experiment on thin film aluminium samples.

The thin film targets were mounted on a computer-controlled $\mathrm{x}-\mathrm{y}$ translation stage. The target was rastered about 4 laser diameters between experiments so that each laser-driven shock would propagate into undisturbed material. Experiments have been performed in the above sample orientation, with the shock-driving laser focussed through the glass substrate onto the aluminium film, as well as in the reverse orientation, with the shock-driving laser focussed onto the aluminium film side. The probe pulses observed the free aluminium surface in the orientation shown in Fig. 1 and the aluminium/glass interface in the reverse orientation.
For the frequency-domain interferometry measurements, two probe pulses were focussed to a ca. $300 \mu \mathrm{m}$ diameter spot at an incidence angle $\sim 45^{\circ}$ to the surface normal. The reflected beam was split in half optically and each half was focussed with $\mathrm{x} 8$ magnification onto the entrance slit of a $0.3 \mathrm{~m}$ focal length imaging spectrograph (Acton Corp). The spatially magnified and spectrally dispersed images were recorded using a CCD camera (Photometrics, Ltd.).

The orientation of the spectrometer entrance slit relative to the target, coupled with the beam splitter in the reflected probe pulse beam path and an image rotation in one of the resulting paths, allowed us to produce two spatially-resolved images on the CCD simultaneously. One image was essentially a vertical slice through the shockdriving laser spot (i.e. runs perpendicular to the plane of the probe pulses). The other image was a horizontal slice. Because the probe pulses were incident on the $\mathrm{Al}$ surface at $\sim 45^{\circ}$, the horizontal slice has a time component that covers approximately $1 \mathrm{ps}$. The crossing point of the slices was adjusted optically to the center of the shockdriving laser spot. This dual imaging allows a single experiment to produce spatially-resolved data at a single probe/pump delay time (the vertical slice) as well as a set of data that is a convolution between space and time (the horizontal slice).

The time delay between the two probe pulses and the time delay between the shock-driving laser pulse and the first probe pulse were adjusted with better than 100 fs precision using computer controlled precision translation stages (Newport Corp.).

The two probe pulses are stretched in time by the spectrograph, so that they overlap in time and space at the CCD and produce an interference pattern along the wavelength axis. The resulting CCD image for each experiment was analyzed usng Fourier transform methods, as in Refs. 7 and 8 , to extract the difference in phase between the two probe pulses caused by changes in the film surface position or the optical properties of the aluminium film that occurred in the time between the pulses. 


\section{RESULTS}

Phase shift differences between two probe pulses 8 ps apart measured using frequency-domain interferometry in two thin-film samples are shown in Figure 2. Each sample had a quadrant with 20 $\mathrm{nm}$ thick aluminium film, and the remainder was $960 \mathrm{~nm}$ or $442 \mathrm{~nm}$ thick aluminium, as measured by the supplier. The $20 \mathrm{~nm}$ region allowed essentially a time-zero measurement.

Several things are immediately evident in this data. First, the phase shift increases in nearly a linear fashion from zero to a maximum in about 8 ps. Second, there is a very slight decrease in phase shift just before the steep rise, which is more apparent in the thicker parts of the samples. Third, the maximum phase shift is larger in the $20 \mathrm{~nm}$ sections than in either thicker region, and is very slightly larger in the $442 \mathrm{~nm}$ than the $960 \mathrm{~nm}$ sample. Finally, the shock transit time in each sample can be estimated using the $20 \mathrm{~nm}$ layer as time zero and the onset of the phase shift in the thicker portions.

\section{DISCUSSION}

The phase shift increases linearly from zero to a maximum in $\sim 8$ ps because the two probe pulses were 8 ps apart. If the shock arrives at the $\mathrm{Al}$ surface after both probe pulses, the surface is essentially unchanged, giving zero phase shift. If the shock arrives between the two probe pulses, the measured phase shift is proportional to the fraction of the time between probe pulses that the surface is moving. If the shock arrives before both probe pulses, a maximum phase shift is reached. The small decrease in phase shift before the steep rise may be due to thermal electron transport ahead of the shock (10). Hydrodynamic modeling indicates that the laser driven shock pressure decreases with distance traveled (unsupported), apparently verified by the decreasing maximum phase shift with increasing sample thickness. The measured shock transit times in the two samples give average shock velocities that are reasonable (ca. $6 \mathrm{~nm} / \mathrm{ps}$ ), and that decrease with sample thickness.

Finally, the curvature at the onset of the phase shift rise gives some indication of the free-surface velocity rise time. All four sets of data show very little curvature in that region, indicating that the rise time is significantly less than $8 \mathrm{ps}$.

\section{ACKNOWLEDGEMENT}

This research was performed under the auspices of the US Department of Energy.

\section{REFERENCES}

1. Barker L.M. and Hollenbach R.E., Rev. Sci. Instrum. 36, 1617 (1065)

2. Durand M., Laharrague P., Lalle P., Le Bihan A., Morvan J., and Pujols H., Rev. Sci. Instrum. 48, 275 (1977)

3. Bloomquist D.D. and Sheffield S.A., J. Appl. Phys. 54, 1717 (1983)

4. Hemsing W.F., in Proceedings of the Eighth Symposium (Intl.) on Detonation, Naval Surface Weapons Center NSWC MP 86-194, 468 (1985)

5. Tas G., Franken J., Hambir S.A., Dlott D.D., Phys. Rev. Lett. 78, 4585 (1997)

6. Tas G., Hambir S.A., Franken J., Hare D.E., Dlott D.D., J. Appl. Phys. 82, 1080 (1997)

7. Tokunaga E., Terasaki A., and Kobayashi T., Opt. Lett. 17, 1131 (1992)

8. Geindre J.P., Audebert P., Rousse A., Falliès F., Gauthier J.C., Mysyrowicz A., Dos Santos A., Hamoniaux G., and Antonetti A., Opt. Lett. 19, 1997 (1994)

9. Evans R., Badger A.D., Failliès F., Mahdieh M., Hall T.A., Audebert P., Geindre J.-P., Gauthier J.-C., Mysyrowicz A., Grillon G., and Antonetti A., Phys. Rev. Lett. 77, 3359 (1996).

10. Ng A., Forsman A., and Chiu G., Phys. Rev. Lett. 81, 2914 (1998). 

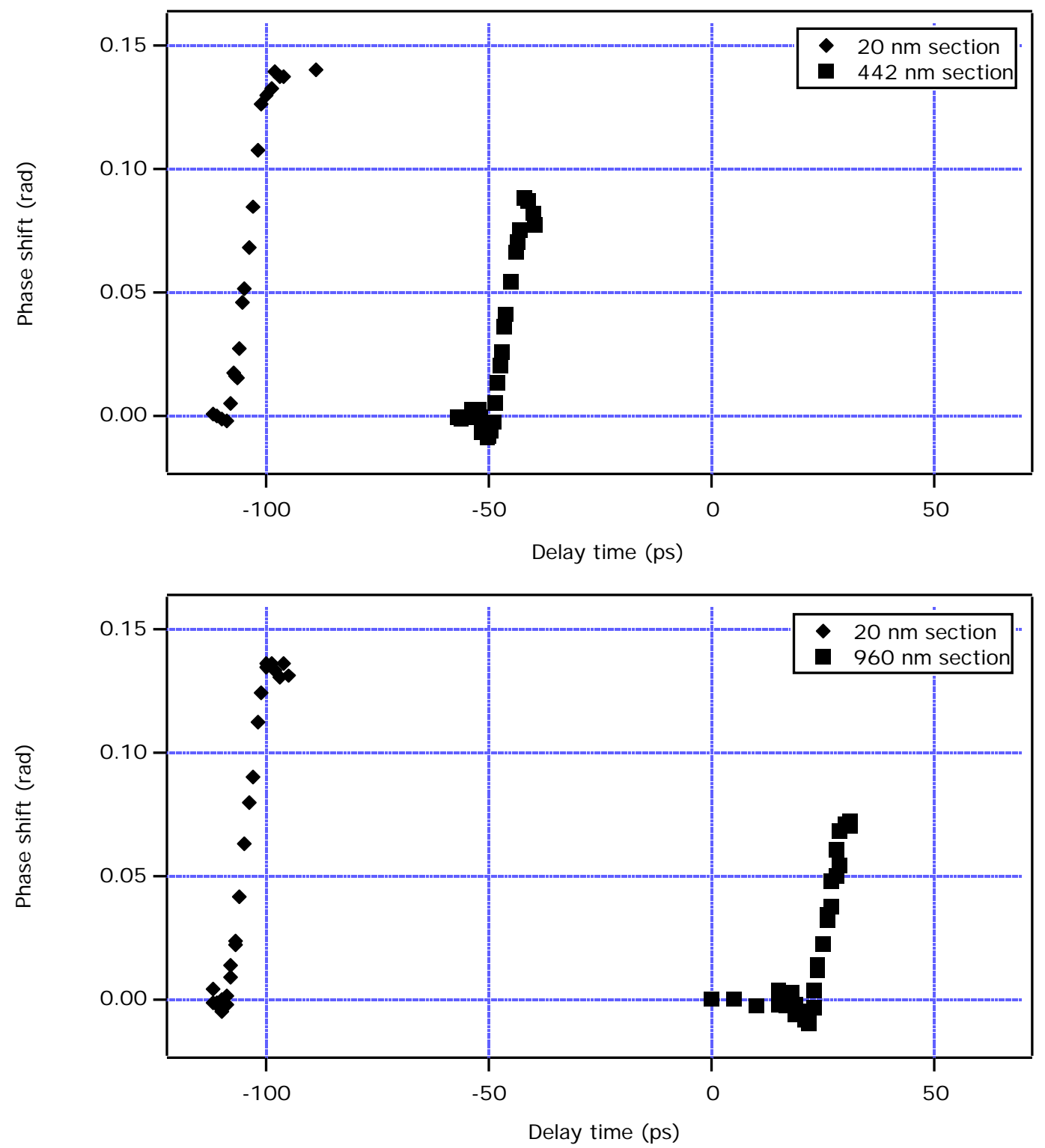

Figure 2: Phase shift (radians) measured using frequency domain interferometry in thin film samples: (top) sample had $20 \mathrm{~nm}$ and $442 \mathrm{~nm}$ sections; (bottom) sample had $20 \mathrm{~nm}$ and $960 \mathrm{~nm}$ sections 